\title{
EXIT-Chart-Aided Near-Capacity Quantum Turbo Code Design
}

\author{
Zunaira Babar, Soon Xin Ng, Senior Member, IEEE, and Lajos Hanzo, Fellow, IEEE
}

\begin{abstract}
4 Abstract-High detection complexity is the main impediment 5 in future gigabit-wireless systems. However, a quantum-based 6 detector is capable of simultaneously detecting hundreds of user 7 signals by virtue of its inherent parallel nature. This, in turn, 8 requires near-capacity quantum error correction codes for pro9 tecting the constituent qubits of the quantum detector against 10 undesirable environmental decoherence. In this quest, we appro11 priately adapt the conventional nonbinary EXtrinsic Information 12 Transfer (EXIT) charts for quantum turbo codes (QTCs) by 13 exploiting the intrinsic quantum-to-classical isomorphism. The 14 EXIT chart analysis not only allows us to dispense with the 15 time-consuming Monte Carlo simulations but facilitates the design 16 of near-capacity codes without resorting to the analysis of their 17 distance spectra as well. We have demonstrated that our EXIT 18 chart predictions are in line with the Monte Carlo simulation 19 results. We have also optimized the entanglement-assisted QTC 20 using EXIT charts, which outperforms the existing distance-spec21 tra-based QTCs. More explicitly, the performance of our opti22 mized QTC is as close as $0.3 \mathrm{~dB}$ to the corresponding hashing 23 bound.
\end{abstract}

24 Index Terms-EXtrinsic Information Transfer (EXIT) charts, 25 near-capacity design, quantum error correction, turbo codes.

\section{INTRODUCTION}

27 ULTIUSER multiple-input multiple-output (MU28 ( MIMO) [1], [2] and massive MIMO [3] schemes 29 are promising candidates for the future-generation gigabit30 wireless system. However, the corresponding detection 31 complexity exponentially increases with the number of users 32 and antennas, when aiming for approaching the optimum 33 maximum-likelihood (ML) performance. An attractive solution 34 to this exponentially escalating complexity problem is to 35 perform the ML detection in the quantum domain, since 36 quantum computing allows parallel evaluations of a function 37 at a complexity cost that is equivalent to a single classical 38 evaluation [4], [5]. However, a quantum detector requires 39 powerful quantum error correction codes (QECCs) for 40 stabilizing and protecting the fragile constituent quantum

Manuscript received December 14, 2013; revised April 22, 2014; accepted May 31, 2014. This work was supported in part by the Engineering and Physical Sciences Research Council under Grant EP/L018659/1; by the Research Councils UK under the India-UK Advanced Technology Center; by the EU under the CONCERTO project; by the European Research Council, Advanced Fellow Grant; and by the Royal Society's Wolfson Reasearch Merit Award. The review of this paper was coordinated by Prof. H. H. Nguyen.

The authors are with the School of Electronics and Computer Science, University of Southampton, Southampton SO17 1BJ, U.K. (e-mail: zb2g10@ ecs.soton.ac.uk; sxn@ecs.soton.ac.uk; lh@ecs.soton.ac.uk).

Color versions of one or more of the figures in this paper are available online at http://ieeexplore.ieee.org.

Digital Object Identifier 10.1109/TVT.2014.2328638 bits (qubits) against undesirable quantum decoherence, when 41 they interact with the environment [4], [6]. Furthermore, 42 quantum-based wireless transmission is capable of supporting 43 secure data dissemination [4], [7], where any 'measurement' 44 or "observation" by an eavesdropper will destroy the quantum 45 entanglement, hence instantly intimating the parties concerned 46 [4]. However, this requires powerful QECCs for the reliable 47 transmission of qubits across the wireless communication 48 channels. Hence, near-capacity QECCs are the vital enabling 49 technique for future generations of wireless systems, which 50 are both reliable and secure and yet operate at an affordable 51 detection complexity.

Classical turbo codes operate almost arbitrarily close to 53 the Shannon limit, which inspired researchers to achieve a 54 comparable near-capacity performance for quantum systems 55 [8]-[12]. In this quest, Poulin et al. developed the theory 56 of quantum turbo codes (QTCs) in [8] and [9], based on 57 the interleaved serial concatenation of quantum convolutional 58 codes (QCCs) [13]-[16], and investigated their behavior on a 59 quantum depolarizing channel. ${ }^{1}$ It was found in [8] and [17] 60 that the constituent QCCs cannot be simultaneously recursive 61 and noncatastrophic. Since the recursive nature of the inner 62 code is essential for ensuring an unbounded minimum distance, 63 whereas the noncatastrophic nature is required to achieve de- 64 coding convergence, the QTCs in [8] and [9] had a bounded 65 minimum distance. More explicitly, the design of Poulin et al. 66 [8], [9] was based on nonrecursive and noncatastrophic con- 67 volutional codes. Later, Wilde and Hseih [10] extended the 68 concept of preshared entanglement to QTCs, which facilitated 69 the design of QTCs having an unbounded minimum distance. 70 Wilde et al. also introduced the notion of extrinsic information 71 to the iterative decoding of QTCs and investigated various code 72 structures in [11].

The search for the optimal components of a QTC has been 74 so far confined to the analysis of the constituent QCC distance 75 spectra, followed by intensive Monte Carlo simulations for 76 determining the convergence threshold of the resultant QTC, 77 as detailed in [9] and [11]. While the distance spectrum dom- 78 inates a turbo code's performance in the bit error rate (BER) 79 floor region, it has a relatively insignificant impact on the 80 convergence properties in the turbo-cliff region [18]. Therefore, 81 having a good distance spectrum does not guarantee having a 82

\footnotetext{
${ }^{1} \mathrm{~A}$ quantum channel can be used for modeling imperfections in quantum hardware, namely, faults resulting from quantum decoherence and quantum gates. Furthermore, a quantum channel can also model quantum-state flips imposed by the transmission medium, including free-space wireless channels and optical fiber links, when qubits are transmitted across these media.
} 
83 near-capacity performance; in fact, there is often a tradeoff 84 between them. To circumvent this problem and to dispense 85 with time-consuming Monte Carlo simulations, in this paper, 86 we extend the application of EXtrinsic Information Transfer 87 (EXIT) charts to the design of QTCs.

88 More explicitly, we have the following contributions.

89 - We have appropriately adapted the conventional nonbi90 nary EXIT-chart-based design approach to the family of 91 QTCs based on the underlying quantum-to-classical iso92 morphism. Similar to the classical codes, our EXIT chart 93 predictions are in line with the Monte Carlo simulation 94 results.

95 - We have analyzed the behavior of both an unassisted (non96 recursive) and an entanglement-assisted (recursive) inner 97 convolutional code using EXIT charts for demonstrating 98 that, similar to their classical counterparts, recursive inner 99 quantum codes constitute families of QTCs having an 100 unbounded minimum distance.

101

102

103

104

105

106

107

108

109

110

111

112

113

114 115 rudimentary introduction to quantum stabilizer codes (QSCs) 116 and QTCs. We will then present our proposed EXIT-chart117 based approach conceived for QTCs in Section III. Our results 118 will be discussed in Section IV, and our conclusions are offered 119 in Section V.

\section{PRELIMINARIES}

121 The constituent convolutional codes of a QTC belong to the 122 class of stabilizer codes [19], which are analogous to classical 123 linear block codes. Here, we will briefly review the basics of 124 stabilizer codes to highlight this relationship for the benefit of 125 readers with background in classical channel coding. This will 126 be followed by a brief discussion on QTCs.

\section{A. Stabilizer Codes}

128 Qubits collapse to classical bits upon measurement [5], [6]. 129 This prevents us from directly applying classical error cor130 rection techniques for reliable quantum transmission. QECCs 131 circumvent this problem by observing the error syndromes 132 without reading the actual quantum information. Hence, QSCs 133 invoke the syndrome decoding approach of classical lin134 ear block codes for estimating the errors incurred during 135 transmission.

136 Let us first recall some basic definitions [6].
Pauli Operators: The I, X, Y, and Z Pauli operators are 137 defined by the following matrices:

$$
\begin{gathered}
\mathbf{I}=\left(\begin{array}{ll}
1 & 0 \\
0 & 1
\end{array}\right), \quad \mathbf{X}=\left(\begin{array}{cc}
0 & 1 \\
1 & 0
\end{array}\right) \\
\mathbf{Y}=\left(\begin{array}{cc}
0 & -i \\
i & 0
\end{array}\right), \mathbf{Z}=\left(\begin{array}{cc}
1 & 0 \\
0 & -1
\end{array}\right)
\end{gathered}
$$

where the $\mathbf{X}, \mathbf{Y}$, and $\mathbf{Z}$ operators anticommute with each other. 139

Pauli Group: A single-qubit Pauli group $\mathcal{G}_{1}$ consists of all 140 the Pauli matrices of (1), together with the multiplicative factors 141 \pm 1 and $\pm i$, i.e., we have

$$
\mathcal{G}_{1} \equiv\{ \pm \mathbf{I}, \pm i \mathbf{I}, \pm \mathbf{X}, \pm i \mathbf{X}, \pm \mathbf{Y}, \pm i \mathbf{Y}, \pm \mathbf{Z}, \pm i \mathbf{Z}\}
$$

The general Pauli group $\mathcal{G}_{n}$ is an $n$-fold tensor product of $\mathcal{G}_{1} . \quad 143$

Depolarizing Channel: The depolarizing channel character- 144 ized by probability $p$ inflicts an $n$-tuple error $\mathcal{P} \in \mathcal{G}_{n}$ on $n 145$ qubits, where the $i$ th qubit may experience either a bit flip (X), 146 a phase flip $(\mathbf{Z})$, or both $(\mathbf{Y})$ with a probability of $p / 3$.

An $[n, k]$ QSC, constructed over a code space $\mathcal{C}$, is defined 148 by a set of $(n-k)$ independent commuting $n$-tuple Pauli oper- 149 ators $g_{i}$, for $1 \leq i \leq(n-k)$. The corresponding encoder then 150 maps the information word (logical qubits) $|\psi\rangle \in \mathbb{C}^{2^{k}}$ onto the 151 codeword (physical qubits) $|\bar{\psi}\rangle \in \mathbb{C}^{2^{n}}$, where $\mathbb{C}^{d}$ denotes the $d$ - 152 dimensional Hilbert space. More specifically, the corresponding 153 stabilizer group $\mathcal{H}$ contains both $g_{i}$ and all the products of $g_{i} 154$ for $1 \leq i \leq(n-k)$ and forms an Abelian subgroup of $\mathcal{G}_{n}$. A 155 unique feature of these operators is that they do not change the 156 state of valid codewords, while yielding an eigenvalue of -1157 for corrupted states. Consequently, the eigenvalue is -1 if the 158 $n$-tuple Pauli error $\mathcal{P}$ anticommutes with the stabilizer $g_{i}$, and 159 it is +1 if $\mathcal{P}$ commutes with $g_{i}$. More explicitly, we have

$$
g_{i}|\hat{\psi}\rangle= \begin{cases}|\bar{\psi}\rangle, & g_{i} \mathcal{P}=\mathcal{P} g_{i} \\ -|\bar{\psi}\rangle, & g_{i} \mathcal{P}=-\mathcal{P} g_{i}\end{cases}
$$

where $\mathcal{P}$ is an $n$-tuple Pauli error, and $|\bar{\psi}\rangle \in \mathcal{C}$ and $|\hat{\psi}\rangle=\mathcal{P}|\bar{\psi}\rangle 161$ is the received codeword. The resultant \pm 1 eigenvalue gives the 162 corresponding error syndrome, which is 0 for an eigenvalue of 163 +1 and 1 for an eigenvalue of -1 . It must be mentioned here 164 that Pauli errors that differ only by the stabilizer group have 165 the same impact on all the codewords and, therefore, can be 166 corrected by the same recovery operations. This gives quantum 167 codes the intrinsic property of degeneracy [20].

168

As detailed in [21] and [22], QSCs may be characterized 169 in terms of an equivalent binary parity-check matrix notation 170 satisfying the commutativity constraint of stabilizers. This can 171 be exploited for designing quantum codes with the aid of 172 known classical codes. The $(n-k)$ stabilizers of an $[n, k] 173$ stabilizer code can be represented as a concatenation of a pair of 174 $(n-k) \times n$ binary matrices $\mathbf{H}_{z}$ and $\mathbf{H}_{x}$, resulting in the binary 175 parity-check matrix $\mathbf{H}$ as given in the following equation:

$$
\mathbf{H}=\left[\mathbf{H}_{z} \mid \mathbf{H}_{x}\right] .
$$

More explicitly, each row of $\mathbf{H}$ corresponds to a stabilizer of 177 $\mathcal{H}$, so that the $i$ th column of $\mathbf{H}_{z}$ and $\mathbf{H}_{x}$ corresponds to the 178 $i$ th qubit, and a binary 1 at these locations represents a $\mathbf{Z} 179$ 
180 and X Pauli operator, respectively, in the corresponding stabi181 lizer. Moreover, the commutativity requirement of stabilizers is 182 transformed into the orthogonality of rows with respect to the 183 symplectic product defined in [22], as follows:

$$
\mathbf{H}_{z} \mathbf{H}_{x}^{T}+\mathbf{H}_{x} \mathbf{H}_{z}^{T}=0 .
$$

184 Conversely, two classical linear codes $\mathbf{H}_{z}$ and $\mathbf{H}_{x}$ can be used 185 to construct a QSC $\mathbf{H}$ of (4) if $\mathbf{H}_{z}$ and $\mathbf{H}_{x}$ meet the symplectic 186 criterion of (5).

187 In line with this discussion, a Pauli error operator $\mathcal{P}$ can be 188 represented by the effective error $P$, which is a binary vector 189 of length $2 n$. More specifically, $P$ is a concatenation of $n$ bits 190 for $\mathbf{Z}$ errors, followed by another $n$ bits for $\mathbf{X}$ errors, and the 191 resultant syndrome is given by the symplectic product of $\mathbf{H}$ 192 and $P$, which is equivalent to $\mathbf{H}\left[P_{x}: P_{z}\right]^{T}$. In other words, 193 the Pauli- $\mathbf{X}$ operator is used for correcting $\mathbf{Z}$ errors, whereas 194 the Pauli-Z operator is used for correcting $\mathbf{X}$ errors [6]. Thus, 195 the quantum-domain syndrome is equivalent to the classical196 domain binary syndrome, and a basic quantum-domain decod197 ing procedure is similar to the syndrome-based decoding of the 198 equivalent classical code [22]. However, due to the degenerate 199 nature of quantum codes, quantum decoding aims at finding the 200 most likely error coset, whereas classical syndrome decoding 201 finds the most likely error.

\section{B. QTCs}

203 Analogous to classical serially concatenated (SC) turbo 204 codes, QTCs are obtained from the interleaved serial concate205 nation of QCCs, which belong to the class of stabilizer codes. 206 However, it is more convenient to exploit the circuit-based 207 representation of the constituent codes, rather than the con208 ventional parity-check-matrix-based syndrome decoding [23]. 209 Before proceeding with the decoding algorithm, we will briefly 210 review the circuit-based representation. This discussion is based 211 on [9].

212 Let us consider an $(n, k)$ classical linear block code con213 structed over the code space $C$, which maps the information 214 word $c \in \mathbb{F}_{2}^{k}$ onto the corresponding codeword $\bar{c} \in \mathbb{F}_{2}^{n}$. In the 215 circuit-based representation, code space $C$ is defined as follows:

$$
C=\left\{\bar{c}=\left(c: 0_{n-k}\right) V\right\}
$$

216 where $V$ is an $(n \times n)$-element invertible encoding matrix over $217 \mathbb{F}_{2}$. Similarly, for an $[n, k]$ QSC, the quantum code space $\mathcal{C}$ is 218 defined as

$$
\mathcal{C}=\left\{|\bar{\psi}\rangle=\mathcal{V}\left(|\psi\rangle \otimes\left|0_{n-k}\right\rangle\right)\right\}
$$

219 where $\mathcal{V}$ is an $n$-qubit Clifford transformation ${ }^{2}$ and $\mid \psi\left\langle\in \mathbb{C}^{2^{k}}\right.$. 220 The corresponding binary encoding matrix $V$ is a unique $(2 n \times$ $2212 n$ )-element matrix such that for any $\mathcal{P} \in \mathcal{G}_{n}$, we have [9]

$$
\left[\mathcal{V P V} \mathcal{V}^{\dagger}\right]=[\mathcal{P}] V
$$

\footnotetext{
${ }^{2}$ Clifford transformation $\mathcal{V}$ is a unitary transformation, which maps an $n$-qubit Pauli group $\mathcal{G}_{n}$ onto itself under conjugation [24], i.e.,
}

$$
\mathcal{V G}_{n} \mathcal{V}^{\dagger}=\mathcal{G}_{n}
$$

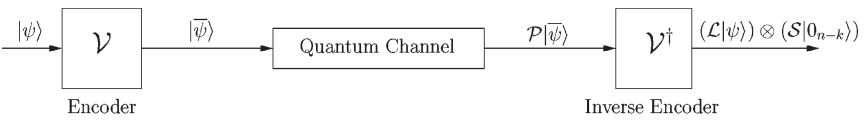

Fig. 1. Quantum transmission model.

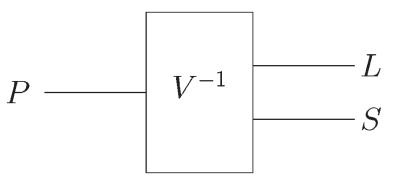

Fig. 2. Circuit representation of the inverse encoder $P V^{-1}=(L: S)$.

where $[\mathcal{P}]=P$, and [.] denotes the effective Pauli group $G_{n} 222$ such that $[\mathcal{P}]$ differs from $\mathcal{P}$ by a multiplicative constant, i.e., 223 we have $[\mathcal{P}]=\mathcal{P} /\{ \pm 1, \pm i\}$. The rows of $V$, which are denoted 224 as $V_{i}$ for $1 \leq i \leq 2 n$, are given by $V_{i}=\left[\mathcal{V} Z_{i} \mathcal{V}^{\dagger}\right]=\left[Z_{i}\right] V$ for 225 $1 \leq i \leq n$ and $V_{i}=\left[\mathcal{V} X_{i} \mathcal{V}^{\dagger}\right]=\left[X_{i}\right] V$ for $n<i \leq 2 n$. Here, 226 $X_{i}$ and $Z_{i}$ represent the Pauli $\mathbf{X}$ and $\mathbf{Z}$ operators acting on 227 the $i$ th qubit. Furthermore, any codeword in $\mathcal{C}$ is invariant 228 by $\mathcal{V} Z_{i} \mathcal{V}^{\dagger}$, for $k<i \leq n$, which therefore corresponds to the 229 stabilizer generators $g_{i}$ of (3). More explicitly, the rows $V_{i}$, for 230 $k<i \leq n$, constitute the $(n-k) \times 2 n$ parity-check matrix $\mathbf{H} 231$ of (4), which meets the symplectic criterion of (5).

232

At the decoder, the received codeword $|\hat{\psi}\rangle=\mathcal{P}|\bar{\psi}\rangle$ is passed 233 through the inverse encoder $\mathcal{V}^{\dagger}$, which yields the corrupted 234 transmitted information word $\mathcal{L}|\psi\rangle$ and the associated syn- 235 drome $\mathcal{S}\left|0_{n-k}\right\rangle$, which is formulated as

$$
\begin{aligned}
\mathcal{V}^{\dagger} \mathcal{P}|\bar{\psi}\rangle & =\mathcal{V}^{\dagger} \mathcal{P} \mathcal{V}\left(|\psi\rangle \otimes\left|0_{n-k}\right\rangle\right) \\
& =(\mathcal{L}|\psi\rangle) \otimes\left(\mathcal{S}\left|0_{n-k}\right\rangle\right)
\end{aligned}
$$

where $\mathcal{L}$ denotes the error imposed on the logical qubits, 237 whereas $\mathcal{S}$ represents the error inflicted on the remaining $(n-238$ $k)$ qubits. This transmission process is summarized in Fig. 1. 239

Since stabilizer codes are analogous to linear block codes, 240 syndrome decoding is employed at the receiver to find the most 241 likely error coset $\mathcal{L}$ given the syndrome $\mathcal{S}$. This is efficiently 242 achieved by exploiting the equivalent binary encoding matrix 243 $V$ of (8), which decomposes the effective $n$-qubit error imposed 244 on the physical qubits $P=[\mathcal{P}]$ into the effective $k$-qubit error 245 inflicted on the logical qubits $L=[\mathcal{L}]$ and the corresponding ef- 246 fective $(n-k)$-qubit syndrome $S=[\mathcal{S}]$, as portrayed in Fig. 2, 247 and mathematically represented as

$$
P V^{-1}=(L: S) .
$$

More explicitly, $P \in G_{n}, L \in G_{k}$, and $S \in G_{n-k}$.

For an $[n, k, m]$ QCC, the encoding matrix $V$ is constructed 250 from repeated use of the seed transformation $U$ shifted by 251 $n$ qubits, as shown in [9, Fig. 6]. More specifically, $U$ is 252 the binary equivalent of an $(n+m)$-qubit symplectic matrix. 253 Furthermore, (10) may be modified as follows [9]:

$$
\left(P_{t}: M_{t}\right) U^{-1}=\left(M_{t-1}: L_{t}: S_{t}\right)
$$

where $t$ and $(t-1)$ denote the current and previous time 255 instants, respectively, whereas $M$ is the effective $m$-qubit 256 error on the memory states. Furthermore, $2(n-k)$-element 257 binary vector $S$ of (10) and (11) can be decomposed into two 258 


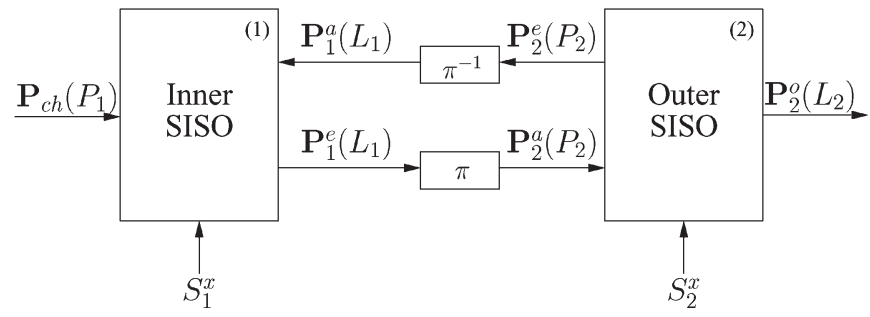

Fig. 3. Schematic of the quantum turbo decoder. $\mathbf{P}_{i}^{a}(),. \mathbf{P}_{i}^{e}($.$) , and \mathbf{P}_{i}^{o}($.$) are$ the a priori, extrinsic, and a posteriori probabilities related to the $i$ th decoder; $P_{i}$ and $L_{i}$ denote the error on the physical and logical qubits, whereas $S_{i}^{x}$ represents the syndrome sequence for the $i$ th decoder.

259 components, yielding $S=S^{x}+S^{z}$, where $S^{x}$ and $S^{z}$ are 260 the $\mathbf{X}$ and $\mathbf{Z}$ components of syndrome $S$, respectively. The $261(n-k)$-binary error syndrome computed using the parity262 check matrix $\mathbf{H}$ only reveals $S^{x}$ but not $S^{z}$ [9]. Therefore, 263 those physical errors that only differ in $S^{z}$ do not have to 264 be differentiated, since they correspond to the same logical 265 error $L$ and can be corrected by the same operations. These 266 are the degenerate errors, which only differ by the stabilizer 267 group, as discussed in Section II-A. Consequently, a quantum 268 turbo decoding algorithm aims at finding the most likely error 269 coset acting on the logical qubits, i.e., $L$, which satisfies the 270 syndrome $S^{x}$.

271 Similar to the classical turbo codes, quantum turbo decoding 272 invokes an iterative decoding algorithm at the receiver for 273 exchanging extrinsic information [11], [25] between the pair of 274 SC soft-in soft-out (SISO) decoders, as shown in Fig. 3. These 275 SISO decoders employ the degenerate decoding approach in 276 [9]. Let $P_{i}$ and $L_{i}$ denote the error imposed on the physical and 277 logical qubits, whereas $S_{i}^{x}$ represents the syndrome sequence 278 for the $i$ th decoder. Furthermore, $\mathbf{P}_{i}^{a}(),. \mathbf{P}_{i}^{e}($.$) , and \mathbf{P}_{i}^{o}($. 279 denote the a priori, extrinsic, and a posteriori probabilities [25] 280 related to the $i$ th decoder. Based on this notation, the turbo 281 decoding process can be summarized as follows.

- The inner SISO decoder uses the channel information $\mathbf{P}_{\mathrm{ch}}\left(P_{1}\right)$, the a priori information gleaned from the outer decoder $\mathbf{P}_{1}^{a}\left(L_{1}\right)$ (initialized to be equiprobable for the first iteration), and the syndrome $S_{1}^{x}$ to compute the extrinsic information $\mathbf{P}_{1}^{e}\left(L_{1}\right)$.

- $\mathbf{P}_{1}^{e}\left(L_{1}\right)$ is passed through a quantum interleaver ${ }^{3}(\pi)$ to yield a priori information for the outer decoder $\mathbf{P}_{2}^{a}\left(P_{2}\right)$.

- Based on the a priori information $\mathbf{P}_{2}^{a}\left(P_{2}\right)$ and on the syndrome $S_{2}^{x}$, the outer SISO decoder computes both the a posteriori information $\mathbf{P}_{2}^{o}\left(L_{2}\right)$ and the extrinsic information $\mathbf{P}_{2}^{e}\left(P_{2}\right)$.

- $\mathbf{P}_{2}^{e}\left(P_{2}\right)$ is deinterleaved to obtain $\mathbf{P}_{1}^{a}\left(L_{1}\right)$, which is fed back to the inner SISO decoder. This iterative procedure continues until convergence is achieved or the maximum affordable number of iterations is reached.

- Finally, a qubit-based MAP decision is made to determine the most likely error coset $L_{2}$.

\footnotetext{
${ }^{3}$ An $N$-qubit quantum interleaver is an $N$-qubit symplectic transformation, which randomly permutes the $N$ qubits and applies single-qubit symplectic transformations to the individual qubits [9].
}

\section{APPLICATION OF EXTRINSIC INFORMATION TRANSFER 299 Charts to Quantum Turbo Codes

Here, we will extend the application of EXIT charts to the 301 quantum domain, by appropriately adapting the conventional 302 nonbinary EXIT chart generation technique to the circuit-based 303 quantum syndrome decoding approach. Some of the informa- 304 tion presented here might seem redundant to the experts of 305 classical channel coding theory. However, since EXIT charts 306 are not widely known in the quantum community, this introduc- 307 tion was necessary to make this treatise accessible to quantum 308 researchers.

\section{A. EXIT Charts}

EXIT charts [18], [25], [26] are capable of visualizing the 311 convergence behavior of iterative decoding schemes by ex- 312 ploiting the input/output relations of the constituent decoders 313 in terms of their average mutual information (MI) characteris- 314 tics. They have been extensively employed for designing near- 315 capacity classical codes [27], [28], [29]. Let us recall that the 316 EXIT chart of an SC scheme visualizes the exchange of the 317 following four MI terms:

- average a priori $\mathrm{MI}$ of the inner decoder, $I_{A}^{1}$;

- average a priori $\mathrm{MI}$ of the outer decoder, $I_{A}^{2}$;

- average extrinsic MI of the inner decoder, $I_{E}^{1}$;

- average extrinsic MI of the outer decoder, $I_{E}^{2}$.

More specifically, $I_{A}^{1}$ and $I_{E}^{1}$ constitute the EXIT curve of 323 the inner decoder, whereas $I_{A}^{2}$ and $I_{E}^{2}$ yield the EXIT curve 324 of the outer decoder. The MI transfer characteristics of both 325 the decoders are plotted in the same graph, with the $x$ - and 326 $y$-axes of the outer decoder swapped. The resultant EXIT chart 327 quantifies the improvement in the MI as the iterations proceed, 328 which can be viewed as a staircase-shaped decoding trajectory. 329 Having an open tunnel between the two EXIT curves ensures 330 that the decoding trajectory reaches the $(1, y)$ point of perfect 331 convergence.

\section{B. Quantum-to-Classical Isomorphism}

Before proceeding with the application of EXIT charts for 334 quantum codes, let us elaborate on the quantum-to-classical 335 isomorphism encapsulated in (4), which forms the basis of 336 our EXIT-chart-aided approach. As discussed in Section II-A, 337 a Pauli error operator $\mathcal{P}$ experienced by an $N$-qubit frame 338 transmitted over a depolarizing channel can be modeled by an 339 effective error vector $P$, which is a binary vector of length $2 N .340$ The first $N$ bits of $P$ denote $Z$ errors, whereas the remaining $N 341$ bits represent $\mathbf{X}$ errors, as shown in Fig. 4. More explicitly, an 342 $\mathbf{X}$ error imposed on the first qubit will yield a 0 and a 1 at the 343 first and $(N+1)$ th index of $P$, respectively. Similarly, a $\mathbf{Z}$ error 344 imposed on the first qubit will give a 1 and a 0 at the first and 345 $(N+1)$ th index of $P$, respectively, whereas a $Y$ error on the 346 first qubit will result in a 1 at both the first and $(N+1)$ th index 347 of $P$. Since a depolarizing channel characterized by probability 348 $p$ incurs $\mathbf{X}, \mathbf{Y}$ and $\mathbf{Z}$ errors with an equal probability of $p / 3,349$ the effective error vector $P$ reduces to two binary symmetric 350 channels (BSCs) having a crossover probability of $2 p / 3$, where 351 we have one channel for the $\mathbf{Z}$ errors and the other for the 352 


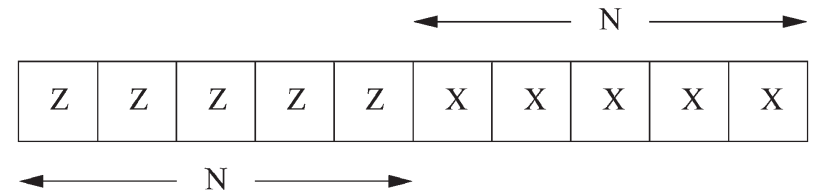

Fig. 4. Effective error $(P)$ corresponding to the error imposed on an $N$-qubit frame $(\mathcal{P})$.

TABLE I

Correlation Between $\mathbf{X}$ and $\mathbf{Z}$ ERrors on the $i$ Th Qubit IN TERMS OF THE CORRESPONDING PROBABILITY OF OCCURRENCE

\begin{tabular}{l|c|c}
\hline & $\mathbf{Z}=0$ & $\mathbf{Z}=1$ \\
\hline $\mathbf{X}=0$ & $1-p$ & $p / 3$ \\
$\mathbf{X}=1$ & $p / 3$ & $p / 3$ \\
\hline
\end{tabular}

$353 \mathbf{X}$ errors. Hence, a quantum depolarizing channel has been 354 considered analogous to a BSC [22], [30], whose capacity is 355 given by

$$
C_{\mathrm{BSC}}=1-H_{2}(2 p / 3)
$$

356 where $\mathrm{H}_{2}$ is the binary entropy function. Using (4), we can 357 readily infer that the code rate $R_{Q}$ of an $[n, k]$ QSC is related to 358 the equivalent classical code rate $R_{C}$ as follows [22], [31]:

$$
R_{C}=\frac{1}{2}\left(1+R_{Q}\right)
$$

359 Consequently, the corresponding quantum capacity is as fol360 lows [22], [31]:

$$
C_{\mathrm{BSC}}^{Q}=1-H_{2}(2 p / 3)
$$

361

362 However, the two BSCs constituting a quantum depolarizing 363 channel are not entirely independent. There is an inherent 364 correlation between the $\mathbf{X}$ and $\mathbf{Z}$ errors [22], which is charac365 terized in Table I. This correlation is taken into account by the 366 turbo decoder in Fig. 3. Alternatively, a quantum depolarization 367 channel can also be considered equivalent to a 4-ary symmetric 368 channel. More explicitly, the $i$ th and the $(N+i)$ th index of $369 P$ constitute the 4 -ary symbol. The corresponding classical 370 capacity is equivalent to the maximum rate achievable over each 371 half of the 4-ary symmetric channel, as follows [22], [31]:

$$
C_{4-\text { ary }}=\frac{1}{2}\left[2-H_{2}(p)-p \log _{2}(3)\right] .
$$

372 Therefore, using (13), the corresponding quantum capacity can 373 be readily shown to be [22], [31]

$$
C_{4-\text { ary }}^{Q}=1-H_{2}(p)-p \log _{2}(3)
$$

374 which is known as the hashing bound. ${ }^{4}$

375 Recall that a quantum code is equivalent to a classical code 376 through (4). More specifically, as mentioned in Section II-A, 377 the decoding of a quantum code is essentially carried out 378 with the aid of the equivalent classical code by exploiting 379 the additional property of degeneracy. Quantum codes employ

\footnotetext{
${ }^{4}$ The hashing bound determines the code rate at which a random quantum code facilitates reliable transmission for a particular depolarizing probability $p[11]$.
}

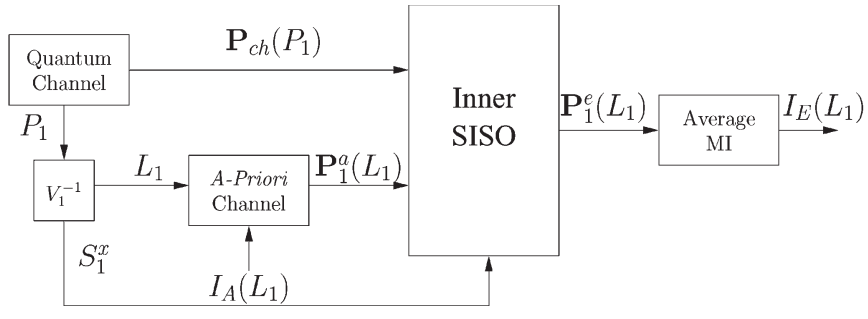

Fig. 5. System model for generating the EXIT chart of the inner decoder.

syndrome decoding [23], which yields information about the 380 error sequence rather than the information sequence or coded 381 qubits, hence avoiding the observation of the latter sequences, 382 which would collapse them back to the classical domain. 383

Since a depolarizing channel is analogous to the BSC and 384 a QTC has an equivalent classical representation, we employ 385 the EXIT chart technique to design near-capacity QTCs. The 386 major difference between the EXIT charts conceived for the 387 classical and quantum domains is that while the former models 388 the a priori information concerning the input bits of the inner 389 encoder (and, similarly, the output bits of the outer encoder), 390 the latter models the a priori information concerning the corre- 391 sponding error sequence, i.e., the error sequence related to the 392 input qubits of the inner encoder $L_{1}$ (and, similarly, the error 393 sequence related to the output qubits of the outer encoder $P_{2}$ ). 394 This will be dealt with further in the following section.

\section{EXIT Charts for QTCs}

Similar to the classical EXIT charts, in our design, we 397 assume that the interleaver length is sufficiently high to ensure 398 that [18], [25]

- the a priori values are fairly uncorrelated;

- the a priori information has a Gaussian distribution.

401

Fig. 5 shows the system model used for generating the 402 EXIT chart of the inner decoder. Here, a quantum depolarizing 403 channel having a depolarizing probability of $p$ generates the 404 error sequence $P_{1}$, which is passed through the inverse inner 405 encoder $V_{1}^{-1}$. This yields both the error imposed on the logical 406 qubits $L_{1}$ and the syndrome $S_{1}^{x}$ according to (10). The a priori 407 channel block then models the a priori information $\mathbf{P}_{1}^{a}\left(L_{1}\right) 408$ such that the average MI between the actual error $L_{1}$ and 409 the a priori probabilities $\mathbf{P}_{1}^{a}\left(L_{1}\right)$ is given by $I_{A}\left(L_{1}\right)$ [18], 410 [25], [26]. More explicitly, we have $I_{A}\left(L_{1}\right)=I\left[L_{1}, \mathbf{P}_{1}^{a}\left(L_{1}\right)\right], 411$ where $I$ denotes the average MI function. As discussed in 412 Section III-B, the $i$ th and $(N+i)$ th bits of the effective error 413 vector $L_{1}$ can be visualized as 4-ary symbols. Consequently, 414 similar to classical nonbinary EXIT charts [32], [33], the 415 a priori information is modeled using an independent Gaussian 416 distribution with zero mean and variance $\sigma_{A}^{2}$, assuming that the 417 $\mathbf{X}$ and $\mathbf{Z}$ errors constituting the 4-ary symbols are independent. 418 Using the channel information $\mathbf{P}_{\mathrm{ch}}\left(P_{1}\right)$, syndrome $S_{1}^{x}$ and the 419 a priori information, the inner SISO decoder yields the extrinsic 420 information $\mathbf{P}_{1}^{e}\left(L_{1}\right)$ based on the classic forward-backward 421 recursive coefficients $\alpha_{t}$ and $\beta_{t}$ as follows [9]: 422

- For a coded sequence of duration $N$, let $P_{1}=\left[P_{1,1}, P_{1,2}, 423\right.$ $\left.\ldots, P_{1, t}, \ldots, P_{1, N}\right]$ and $L_{1}=\left[L_{1,1}, L_{1,2}, \ldots, L_{1, t}, \ldots, L_{1, N}\right], 424$ where $P_{1, t} \in G_{n}$, and $L_{1, t} \in G_{k}$. More explicitly, 425 
$P_{1, t}=\left[P_{1, t}^{1}, P_{1, t}^{2}, \ldots, P_{1, t}^{n}\right]$, and $L_{1, t}=\left[L_{1, t}^{1}, L_{1, t}^{2}, \ldots, L_{1, t}^{k}\right]$. For ease of clarification, we will ignore the first subscript, which represents the decoder, in the algorithm given below, i.e., we have $P_{1}=P$ and $L_{1}=L$. Similarly, $S_{1}^{x}=S^{x}$.

- Let $U=\left(U_{P}: U_{M}\right)$ so that $U_{P}$ is the binary matrix formed by the first $2 n$ columns of $U$ of (11), whereas $U_{M}$ is the binary matrix formed by the last $2 m$ columns of $U$. Therefore, we have

$$
\begin{aligned}
P_{t} & =\left(M_{t-1}: L_{t}: S_{t}\right) U_{P} \\
M_{t} & =\left(M_{t-1}: L_{t}: S_{t}\right) U_{M} .
\end{aligned}
$$

- Let $\alpha_{t}\left(M_{t}\right)$ be the forward recursive coefficient, which is defined as follows:

$$
\begin{aligned}
\alpha_{t}\left(M_{t}\right) & \triangleq \mathbf{P}\left(M_{t} \mid S_{\leq t}^{x}\right) \\
& \propto \sum_{\mu, \lambda, \sigma} \mathbf{P}^{a}\left(L_{t}=\lambda\right) \mathbf{P}_{\mathrm{ch}}\left(P_{t}\right) \alpha_{t-1}(\mu)
\end{aligned}
$$

where $\mu \in G_{m}, \lambda \in G_{k}$ and $\sigma \in G_{n-k}$, while $\sigma=\sigma_{x}+$ $\sigma_{z}$, having $\sigma_{x}=S_{t}^{x}$. Furthermore, we have $P_{t}=(\mu: \lambda$ : $\sigma) U_{P}$ and $M_{t}=(\mu: \lambda: \sigma) U_{M}$. The channel information $\mathbf{P}_{\mathrm{ch}}\left(P_{t}\right)$ is computed assuming that each qubit is independently transmitted over a quantum depolarizing channel having a depolarizing probability of $p$, whose channel transition probabilities are given by [9]

$$
\mathbf{P}_{\mathrm{ch}}\left(P_{t}^{i}\right)= \begin{cases}1-p, & \text { if } \mathcal{P}_{t}^{i}=\mathbf{I} \\ p / 3, & \text { if } \mathcal{P}_{t}^{i} \in\{\mathbf{X}, \mathbf{Z}, \mathbf{Y}\}\end{cases}
$$

- Let $\beta_{t}\left(M_{t}\right)$ be the backward recursive coefficient, which is defined as follows:

$$
\begin{aligned}
\beta_{t}\left(M_{t}\right) & \triangleq \mathbf{P}\left(M_{t} \mid S_{>t}^{x}\right) \\
& \propto \sum_{\lambda, \sigma} \mathbf{P}^{a}\left(L_{t}=\lambda\right) \mathbf{P}_{\mathrm{ch}}\left(P_{t+1}\right) \beta_{t+1}\left(M_{t+1}\right)
\end{aligned}
$$

where $P_{t+1}=\left(M_{t}: \lambda: \sigma\right) U_{P}$, and $M_{t+1}=\left(M_{t}: \lambda\right.$ : $\sigma) U_{M}$.

- Finally, we have the a posteriori probability $\mathbf{P}^{o}\left(L_{t}\right)$, which is given by

$$
\begin{aligned}
\mathbf{P}^{o}\left(L_{t}\right) & \triangleq \mathbf{P}\left(L_{t} \mid S^{x}\right) \\
& \propto \sum_{\mu, \sigma} \mathbf{P}^{a}\left(L_{t}\right) \mathbf{P}_{\mathrm{ch}}\left(P_{t}\right) \alpha_{t-1}(\mu) \beta_{t}\left(M_{t}\right)
\end{aligned}
$$

where $P_{t}=\left(\mu: L_{t}: \sigma\right) U_{P}$ and $M_{t}=\left(\mu: L_{t}: \sigma\right) U_{M}$.

- Marginalized probabilities $\mathbf{P}^{o}\left(L_{t}^{j}\right)$ for $j \in\{0, k-1\}$ are then computed from $\mathbf{P}^{o}\left(L_{t}^{j}\right)$, and the a priori information is removed to yield the extrinsic probabilities [11], i.e., we have

$$
\ln \left[\mathbf{P}^{e}\left(L_{t}^{j}\right)\right]=\ln \left[\mathbf{P}^{o}\left(L_{t}^{j}\right)\right]-\ln \left[\mathbf{P}^{a}\left(L_{t}^{j}\right)\right] .
$$

455 Finally, the extrinsic average MI between $L_{1}$ and $\mathbf{P}_{1}^{e}\left(L_{1}\right)$ is 456 computed, i.e., $I_{E}\left(L_{1}\right)=I\left[L_{1}, \mathbf{P}_{1}^{e}\left(L_{1}\right)\right]$. Since the equivalent 457 classical capacity of a quantum channel is given by the capacity

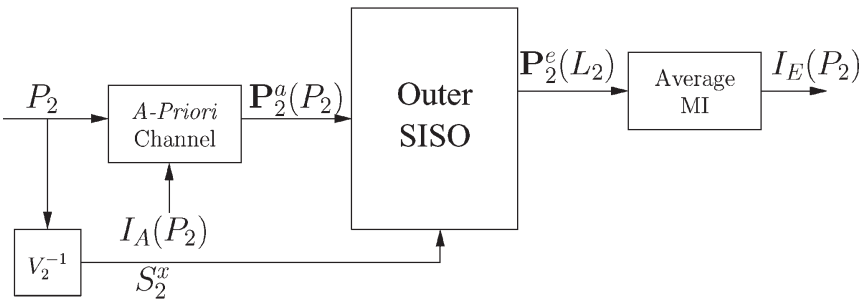

Fig. 6. System model for generating the EXIT chart of the outer decoder.

achievable over each half of the 4-ary symmetric channel as 458 depicted in (15), $I_{E}\left(L_{1}\right)$ is the normalized MI of the 4-ary 459 symbols, which can be computed based on [33] and [34] as 460

$$
I_{E}\left(L_{1}\right)=\frac{1}{2}\left(2+\mathrm{E}\left[\sum_{\mathrm{m}=0}^{3} \mathbf{P}_{1}^{e}\left(L_{1}^{j(\mathrm{~m})}\right) \log _{2} \mathbf{P}_{1}^{e}\left(L_{1}^{j(\mathrm{~m})}\right)\right]\right)
$$

where $E$ is the expectation (or time average) operator, and 461 $L_{1}^{j(\mathrm{~m})}$ is the 4-ary mth hypothetical error imposed on the 462 logical qubits. More explicitly, since the error on each qubit 463 is represented by an equivalent pair of classical bits, $L_{1}^{j(\mathrm{~m})} 464$ is a 4-ary classical symbol with $m \in\{0,3\}$. The process is 465 repeated for a range of $I_{A}\left(L_{1}\right) \in[0,1]$ values for obtaining the 466 extrinsic information transfer characteristics at the depolarizing 467 probability $p$. The resultant inner EXIT function $T_{1}$ of the 468 specific inner decoder may be defined as follows:

469

$$
I_{E}\left(L_{1}\right)=T_{1}\left[I_{A}\left(L_{1}\right), p\right]
$$

which is dependent on the depolarizing probability $p$ of the 470 quantum channel.

The system model used for generating the EXIT chart of 472 the outer decoder is shown in Fig. 6. As inferred from the 473 figure, the EXIT curve of the outer decoder is independent of 474 the channel's output information. The a priori information is 475 generated by the a priori channel based on $P_{2}$ (error on the 476 physical qubits of the second decoder) and $I_{A}\left(P_{2}\right)$, which is 477 the average MI between $P_{2}$ and $\mathbf{P}_{2}^{a}\left(P_{2}\right)$. Furthermore, as for the 478 inner decoder, $P_{2}$ is passed through the inverse outer encoder 479 $V_{2}^{-1}$ to compute $S_{2}^{x}$, which is fed to the outer SISO decoder to 480 yield the extrinsic information $\mathbf{P}_{2}^{e}\left(P_{2}\right)$. Based on (19) and (21), 481 this may be formulated as follows [9]:

$$
\begin{aligned}
\mathbf{P}^{o}\left(P_{t}\right) & \triangleq \mathbf{P}\left(P_{t} \mid S^{x}\right) \\
& \propto \sum_{\mu, \lambda, \sigma} \mathbf{P}\left(P_{t}\right) \mathbf{P}\left(L_{t}=\lambda\right) \alpha_{t-1}(\mu) \beta_{t}\left(M_{t}\right)
\end{aligned}
$$

where $P_{t}=(\mu: \lambda: \sigma) U_{P}$, and $M_{t}=(\mu: \lambda: \sigma) U_{M}$. The re- 483 sultant probabilities are marginalized, and the a priori informa- 484 tion is removed similar to (23). The average MI between $P_{2}$ and 485 $\mathbf{P}_{2}^{e}\left(P_{2}\right)$ is then calculated using (24). The resultant EXIT chart 486 is characterized by the following MI transfer function:

$$
I_{E}\left(P_{2}\right)=T_{2}\left[I_{A}\left(P_{2}\right)\right]
$$

where $T_{2}$ is the outer EXIT function, which is dependent on the 488 specific outer decoder but is independent of the depolarizing 489 probability $p$. 


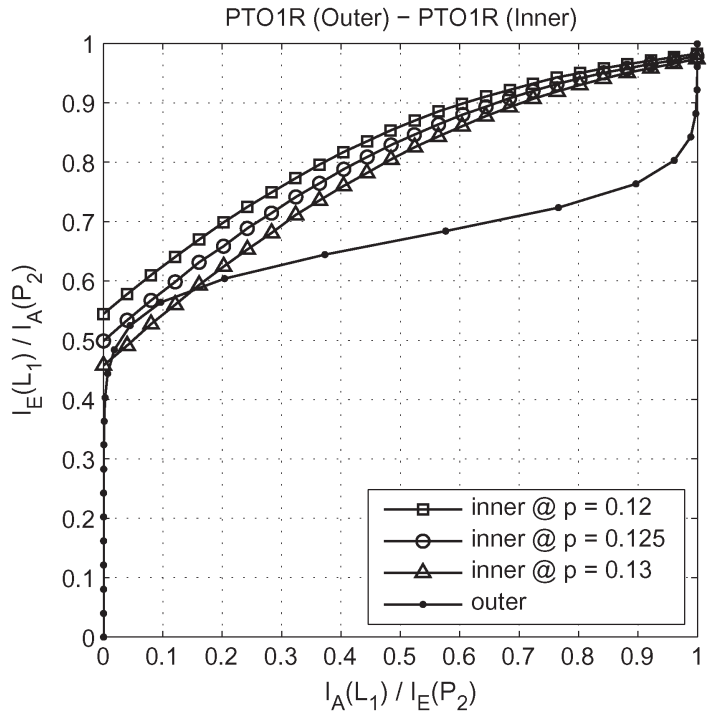

Fig. 7. EXIT curves of a QTC parametrized by the increasing depolarizing probability $p$. Rate-1/9 QTC having PTO1R as both the inner and outer components was used.

491 Finally, the MI transfer characteristics of both decoders 492 characterized by (25) and (27) are plotted in the same graph, 493 with the $x$ - and $y$-axes of the outer decoder swapped.

494

\section{RESULTS AND DisCUSSIONS}

\section{A. Accuracy of EXIT Chart Predictions}

496 To verify the accuracy of our EXIT-chart-based approach, 497 we have analyzed the convergence behavior of a rate-1/9 QTC, 498 consisting of two identical rate-1/3 QCCs. More specifically, 499 for both the inner and outer decoders, we have used the con500 figuration termed as "PTO1R" in [10] and [11], which is a 501 noncatastrophic but quasi-recursive code.

502 Our first aim was to predict the convergence threshold using 503 EXIT charts, which would otherwise require time-consuming 504 word error rate/qubit error rate (WER/QBER) simulations. 505 The convergence threshold can be determined by finding the 506 maximum depolarizing probability $p$, which yields a marginally 507 open EXIT tunnel between the EXIT curves of the inner and 508 outer decoders, hence facilitating an infinitesimally low QBER. 509 Fig. 7 shows the EXIT curves for the inner and outer decoders, 510 where the area under the EXIT curve of the inner decoder 511 decreases upon increasing $p$. Eventually, the inner and outer 512 curves cross over, when $p$ is increased to $p=0.13$. More 513 explicitly, increasing $p$ beyond 0.125 closes the EXIT tunnel. 514 Hence, the convergence threshold is around $p=0.125$.

515 Fig. 8 shows two decoding trajectories superimposed on the 516 EXIT chart in Fig. 7 at $p=0.125$. We have used a 30 000-qubit 517 long interleaver. As shown in Fig. 8, the trajectory successfully 518 reaches the $(x, y)=(1, y)$ point of the EXIT chart. This, in 519 turn, guarantees an infinitesimally low QBER at $p=0.125$ for 520 an interleaver of infinite length.

521 We have further verified the validity of our EXIT chart 522 predictions using QBER simulations. Fig. 9 shows the QBER 523 performance curve for an interleaver length of 3000 qubits. 524 The performance improves upon increasing the number of

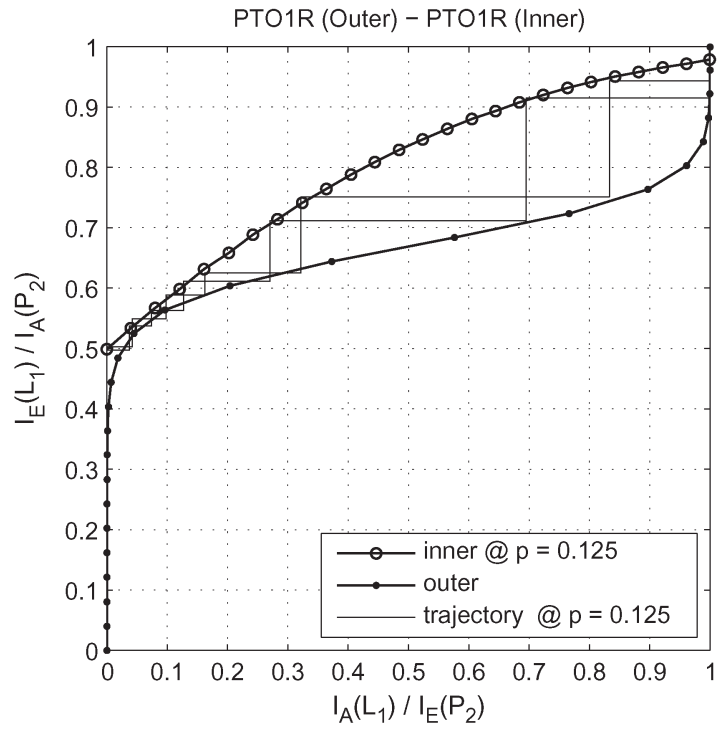

Fig. 8. EXIT chart of a QTC with decoding trajectories at $p=0.125$. Rate-1/9 QTC having PTO1R as both the inner and outer components with an interleaver length of 30000 qubits was used.

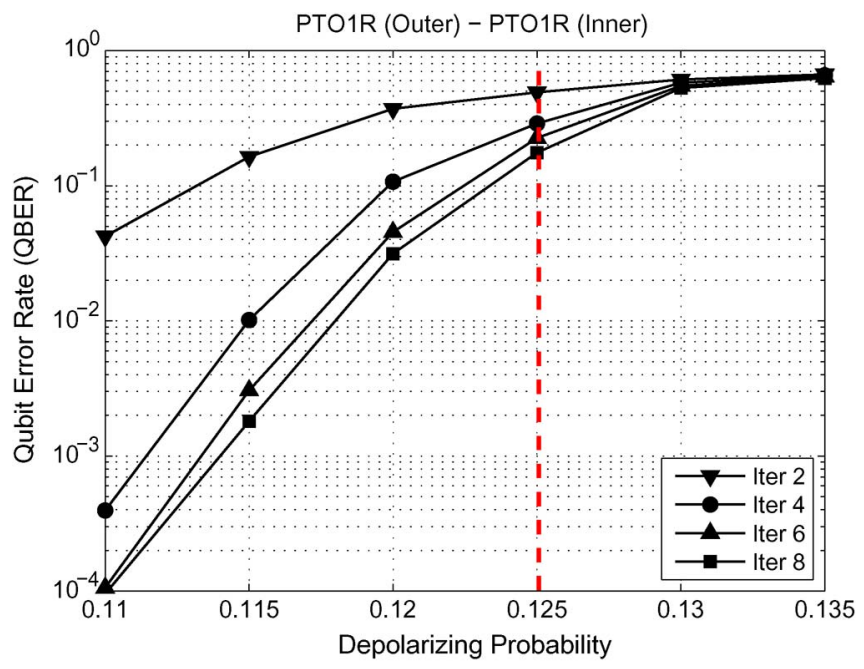

Fig. 9. QBER performance curve with an increasing iteration number for an interleaver length of 3000 qubits. Rate-1/9 QTC having PTO1R as both the inner and outer components was used.

iterations. More specifically, the turbo-cliff region starts around 525 $p=0.125$, whereby the QBER drops as the iterations proceed. 526 Therefore, our EXIT chart predictions closely follow the Monte 527 Carlo simulation results.

\section{B. Entanglement-Assisted and Unassisted Inner Codes}

All noncatastrophic convolutional codes are nonrecursive 530 [9]. Therefore, the resultant families of QTCs have a bounded 531 minimum distance and do not have a true iterative threshold. 532 To circumvent this limitation of QTCs, Wilde et al. [10], [11] 533 proposed to employ entanglement-assisted inner codes, which 534 are recursive and noncatastrophic. The resulting families of 535 entanglement-assisted QTCs have an unbounded minimum dis- 536 tance [10], [11], i.e., their minimum distance increases almost 537 linearly with the interleaver length. Here, we verify this by ana- 538 lyzing the inner decoder's EXIT curves for both the unassisted 539 


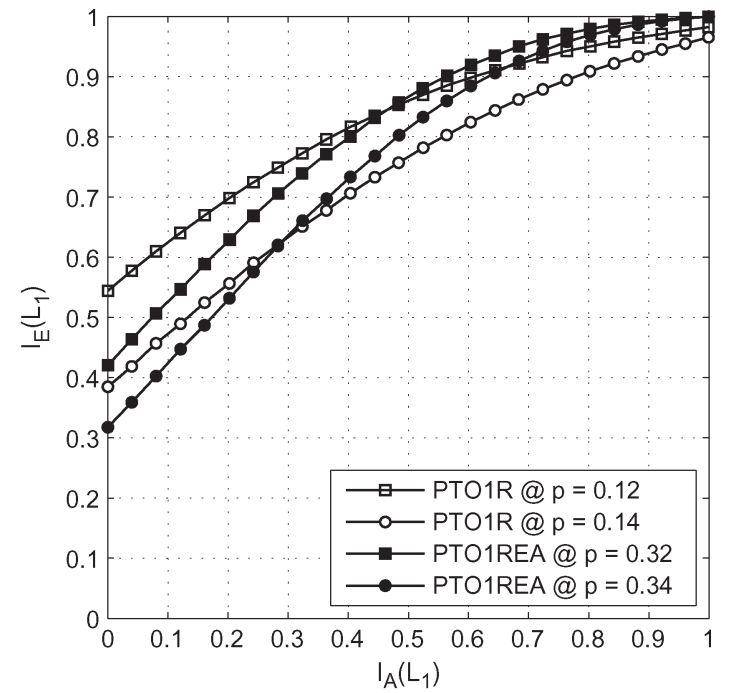

Fig. 10. Comparison of the inner EXIT curves of both unassisted and entanglement-assisted QCCs, which are labeled as PTO1R and PTO1REA, respectively.

540 (nonrecursive) and entanglement-assisted (recursive) inner con541 volutional codes.

542 For classical recursive inner codes, the inner decoder's EXIT 543 curve reaches the $(x, y)=(1,1)$ point, ${ }^{5}$ which guarantees 544 perfect decoding convergence to a vanishingly low QBER 545 as well as having an unbounded minimum distance for the 546 infinite family of QTCs [9] based on these inner codes. Con547 sequently, the resulting families of QTCs have unbounded 548 minimum distance, and hence, an arbitrarily low QBER can 549 be achieved for an infinitely long interleaver. This also holds 550 true for recursive QCCs, as shown in Fig. 10. In this figure, 551 we compare the inner decoder's EXIT curves of both the 552 unassisted and the entanglement-assisted QCCs in [10], which 553 are labeled "PTO1R" and "PTO1REA," respectively. For the 554 PTO1R configuration, decreasing the depolarizing probability 555 from $p=0.14$ to $p=0.12$ shifts the inner decoder's EXIT 556 curve upward and toward the $(1,1)$ point. Hence, the EXIT 557 curve will manage to reach the $(1,1)$ point only at very low 558 values of depolarizing probability. By contrast, the EXIT curve 559 of PTO1REA always terminates at $(1,1)$, regardless of the value 560 of $p$. Therefore, provided that an open EXIT tunnel exists 561 and the interleaver length is sufficiently long, the decoding 562 trajectories of an entanglement-assisted QTC will always reach 563 the $(1,1)$ point, thus guaranteeing an arbitrarily low QBER for 564 the infinite family of QTCs based on these inner codes. In 565 other words, the performance improves upon increasing the 566 interleaver length, thus implying that the minimum distance 567 increases upon increasing the interleaver length, and therefore, 568 the resultant QTCs have an unbounded minimum distance.

\footnotetext{
${ }^{5}$ Note that we only need $(x, y)=(1, y)$ to achieve decoding convergence to an infinitesimally low QBER. However, this requires an outer code having a sufficiently large minimum distance for the sake of ensuring that the outer code's EXIT curve does not intersect with that of the inner code before reaching the $(1, y)$ point. Unfortunately, an outer code having a large minimum distance would result in an EXIT curve having a large open-tunnel area. Thus, it will operate far from the capacity.
}

\section{Optimized QTC Design}

The QTC design in [10] and [11] characterized in Fig. 8570 exhibits a large area between the inner and outer decoder's 571 EXIT curves. The larger the "open-tunnel" area, the farther 572 the QBER performance curve from the achievable capacity 573 limit [25]. Consequently, various distance-spectra-based QTCs 574 investigated in [11] operate within $0.9 \mathrm{~dB}$ of the hashing bound. 575 For the sake of achieving a near-capacity performance, we 576 minimize the area between the inner and outer EXIT curves, 577 so that a narrow but still marginally open tunnel exists at 578 the highest possible depolarizing probability. Our aim was to 579 construct a rate-1/9 QTC relying on an entanglement-assisted 580 inner code (recursive and noncatastrophic) and an unassisted 581 outer code (noncatastrophic) having a memory of 3 and a rate 582 of 1/3. The resultant QTC has an entanglement consumption 583 rate of $6 / 9$, for which the corresponding maximum tolera- 584 ble depolarizing probability was shown to be $p_{\max }=0.3779585$ in [11].

For the sake of designing a near-capacity QTC operating 587 close to the capacity limit of $p_{\max }=0.3779$, we randomly 588 selected both inner and outer encoders from the Clifford group 589 according to the algorithm in [35] to find the inner and outer 590 components, which minimize the area between the correspond- 591 ing EXIT curves. Based on this design criterion, we found 592 an optimal inner and outer code pair whose seed transforms ${ }^{6} 593$ (decimal representation) are given by

$$
\begin{aligned}
U_{\text {inner }}=\{4091,3736,2097,1336,1601,279 & \\
& 3093,502,1792,3020,226,1100\} \\
U_{\text {outer }}=\{1048,3872,3485,2054,983,3164 & \\
& 3145,1824,987,3282,2505,1984\} .
\end{aligned}
$$

Fig. 11 shows the corresponding EXIT chart at the convergence 595 threshold of $p=0.35$. As observed in Fig. 11, a marginally 596 open EXIT tunnel exists between the two curves, which fa- 597 cilitates for the decoding trajectories to reach the $(1,1)$ point. 598 Hence, our optimized QTC has a convergence threshold of $p=599$ 0.35 , which is only $\left[10 \times \log _{10}(0.35 / 0.3779)\right]=0.3 \mathrm{~dB}$ from 600 the maximum tolerable depolarizing probability of 0.3779 . The 601 corresponding QBER performance curves recorded for our op- 602 timized design are given in Fig. 12. A maximum of 15 iterations 603 were used, while the interleaver length was increased from 604 1500 to 12 000. Similar to classical turbo codes, increasing 605 the interleaver length for $p<0.35$ improves the attainable per- 606 formance. Furthermore, Fig. 12 also compares our optimized 607 design with the rate-1/9 QTC in [11] for an interleaver length of 608 3000, which is labeled "PTO1REA-PTO1R" in the figure. For 609 the "PTO1REA-PTO1R" configuration, the turbo-cliff region 610 emerges around 0.31 , which is within $0.9 \mathrm{~dB}$ of the capacity 611 limit. Therefore, our EXIT-chart-based QTC outperforms the 612 QTC design based on the distance spectrum [11]. More specif- 613 ically, the "PTO1REA-PTO1R" configuration yields a QBER 614 of $10^{-3}$ at $p=0.2925$, whereas our optimized QTC gives a 615

\footnotetext{
${ }^{6}$ See [11] for the details of this representation.
} 


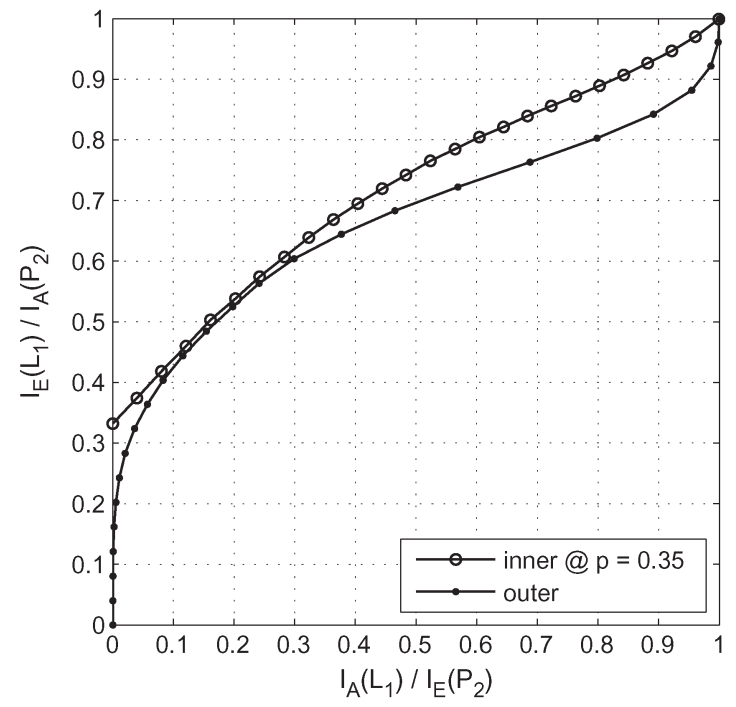

Fig. 11. EXIT chart of the optimized rate-1/9 QTC (Interleaver length $=$ 30000 qubits).

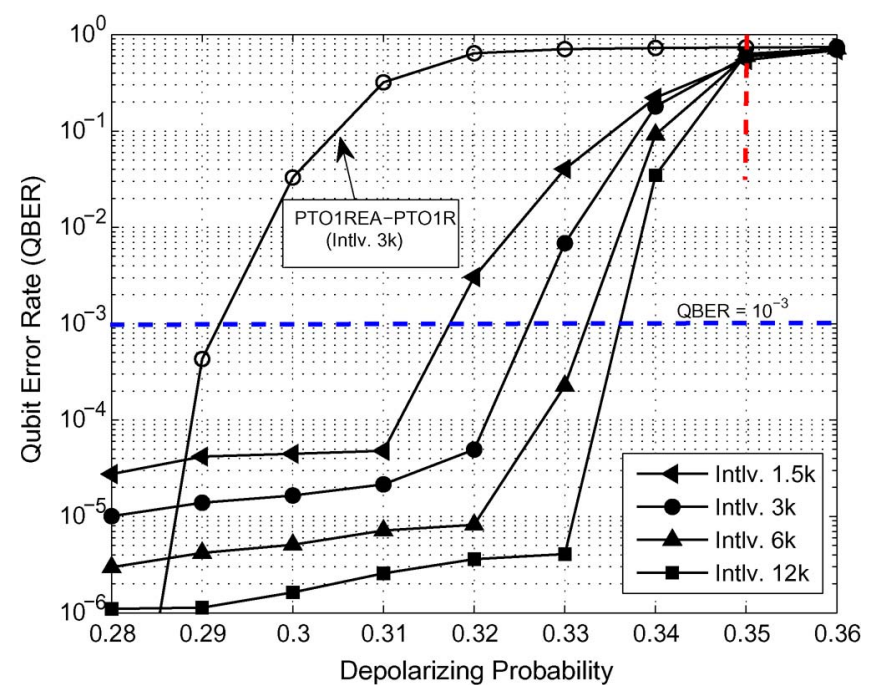

Fig. 12. QBER performance curves of the optimized rate-1/9 QTC for varying interleaver lengths and a maximum of 15 iterations.

616 QBER of $10^{-3}$ at $p=0.3275$. Hence, our optimized QTC 617 outperforms the "PTO1REA-PTO1R" configuration by about $618\left[10 \times \log _{10}(0.2925 / 0.3275)\right]=0.5 \mathrm{~dB}$ at a QBER of $10^{-3}$. 619 However, our main design objective was to not carry out an 620 exhaustive code search but to demonstrate the explicit benefit 621 of our EXIT-chart-based approach in the context of quantum 622 codes. It must also be observed in Fig. 12 that a relatively high 623 error floor exists for our optimized design, which is gradually 624 reduced upon increasing the interleaver length. This is because 625 the outer code has a low minimum distance of only 3. Its 626 truncated distance spectrum is as follows:

$$
\begin{aligned}
D(x)= & 2 x^{3}+19 x^{4}+108 x^{5}+530 x^{6}+2882 x^{7}+14179 x^{8} \\
& +62288 x^{9}+243234 x^{10}+845863 x^{11}+1165784 x^{12} \\
& +2501507 x^{13}+744394 x^{14} .
\end{aligned}
$$

By contrast, the truncated distance spectrum of "PTO1R," 627 which has a minimum distance of 5, is given by [11]

$$
\begin{aligned}
D(x)=11 x^{5}+47 x^{6} & +253 x^{7}+1187 x^{8}+6024 x^{9} \\
& +30529 x^{10}+153051 x^{11}+771650 x^{12} .
\end{aligned}
$$

Consequently, as gleaned from Fig. 12, the "PTO1REA- 629 PTO1R" configuration has a much lower error floor $\left(<10^{-6}\right), 630$ since the outer code "PTO1R" has a higher minimum distance. 631 However, this enlarges the area between the inner and outer 632 decoder's EXIT curves, thus driving the performance farther 633 away from the achievable capacity, as shown in Fig. 8. Hence, 634 there is a tradeoff between the minimization of the error floor 635 and achieving a near-capacity performance. More specifically, 636 while the distance-spectrum-based design primarily aims at 637 achieving a lower error floor, the EXIT-chart-based design 638 strives for achieving a near-capacity performance.

\section{CONCLUSION}

640

In this paper, we have extended the application of classical 641 nonbinary EXIT charts to the circuit-based syndrome decoder 642 of QTCs, to facilitate the EXIT-chart-based design of QTCs. 643 We have verified the accuracy of our EXIT chart generation 644 approach by comparing the convergence threshold predicted 645 by the EXIT chart to the Monte Carlo simulation results. 646 Furthermore, we have shown with the aid of EXIT charts 647 that entanglement-assisted recursive QCCs have an unbounded 648 minimum distance. Moreover, we have designed an optimal 649 entanglement-assisted QTC using EXIT charts, which outper- 650 forms the distance-spectra-based QTC in [11] by about $0.5 \mathrm{~dB} 651$ at a QBER of $10^{-3}$.

\section{ACKNOWLEDGMENT}

653

The authors would like to thank Dr. M. M. Wilde for the 654 valuable discussions and the anonymous reviewers for their 655 insightful advice and comments.

\section{REFERENCES}

[1] S. X. Ng and L. Hanzo, "On the MIMO channel capacity of multidimen- 658 sional signal sets," IEEE Trans. Veh. Technol., vol. 55, no. 2, pp. 528-536, 659 Mar. 2006.

[2] J.-M. Chung, J. Kim, and D. Han, "Multihop hybrid virtual MIMO scheme 661 for wireless sensor networks," IEEE Trans. Veh. Technol., vol. 61, no. 9, 662 pp. 4069-4078, Nov. 2012.

663

[3] J. Lee and S.-H. Lee, "A compressed analog feedback strategy for 664 spatially correlated massive MIMO systems," in Proc. IEEE VTC Fall, 665 2012, pp. 1-6.

[4] S. Imre and F. Balazs, Quantum Computing and Communications: An 667 Engineering Approach. Hoboken, NJ, USA: Wiley, 2005.

668

[5] P. Botsinis, S. X. Ng, and L. Hanzo (2013). Quantum search algo- 669 rithms, quantum wireless, a low-complexity maximum likelihood it- 670 erative quantum multi-user detector design. IEEE Access [Online]. 671 vol. 1, pp. 94-122. Available: http://ieeexplore.ieee.org/xpl/articleDetails. 672 jsp?arnumber $=6515077$

[6] M. A. Nielsen and I. L. Chuang, Quantum Computation and Quantum 674 Information. Cambridge, U.K.: Cambridge Univ. Press, 2000.

[7] X. Zhou and M. McKay, "Secure transmission with artificial noise over 676 fading channels: Achievable rate and optimal power allocation," IEEE 677 Trans. Veh. Technol., vol. 59, no. 8, pp. 3831-3842, Oct. 2010.

[8] D. Poulin, J.-P. Tillich, and H. Ollivier, "Quantum serial turbo-codes," in 679 Proc. IEEE ISIT, Jul. 2008, pp. 310-314.

[9] D. Poulin, J. Tillich, and H. Ollivier, "Quantum serial turbo codes," IEEE 681 Trans. Inf. Theory, vol. 55, no. 6, pp. 2776-2798, Jun. 2009. 
683 [10] M. M. Wilde and M.-H. Hsieh, "Entanglement boosts quantum turbo 684 codes," in Proc. IEEE ISIT, Aug. 2011, pp. 445-449.

685 [11] M. Wilde, M.-H. Hsieh, and Z. Babar, "Entanglement-assisted quantum 686 turbo codes," IEEE Trans. Inf. Theory, vol. 60, no. 2, pp. 1203-1222, 687 Feb. 2014

688 [12] Z. Babar, S. X. Ng, and L. Hanzo, "Near-capacity code design for 689 entanglement-assisted classical communication over quantum depolariz690 ing channels," IEEE Trans. Commun., vol. 61, no. 12, pp. 4801-4807, 691 Dec. 2013

692 [13] H. Ollivier and J.-P. Tillich (2003, Oct.). Description of a quantum con693 volutional code. Phys. Rev. Lett. [Online]. vol. 91, no. 17, pp. $177902-1-$ 694 177902-4. Available: http://link.aps.org/doi/10.1103/PhysRevLett.91. 695177902

696 [14] quant-ph/0401134 H. Ollivier and J. P. Tillich, Quantum Convolutional 697 Codes: Fundamentals 2004, quant-ph/0401134.

698 [15] G. D. Forney, M. Grassl, and S. Guha, "Convolutional and tail-biting 699 quantum error-correcting codes," IEEE Trans. Inf. Theory, vol. 53, no. 3, 700 pp. 865-880, Mar. 2007.

701 [16] M. Grassl and M. Rotteler, "Constructions of quantum convolutional 702 codes," in Proc. IEEE ISIT, Jun. 2007, pp. 816-820.

703 [17] M. Houshmand and M. Wilde, "Recursive quantum convolutional en704 coders are catastrophic: A simple proof," IEEE Trans. Inf. Theory, vol. 59, 705 no. 10, pp. 6724-6731, Oct. 2013.

706 [18] S. ten Brink, "Convergence behavior of iteratively decoded parallel concatenated codes," IEEE Trans. Commun., vol. 49, no. 10, pp. 1727-1737, Oct. 2001.

9] D. Gottesman, "Class of quantum error-correcting codes saturating the quantum Hamming bound," Phys. Rev. A, At. Mol. Opt. Phys., vol. 54, no. 3, pp. 1862-1868, Sep. 1996.

20] E. Pelchat and D. Poulin, "Degenerate Viterbi decoding," IEEE Trans. Inf. Theory, vol. 59, no. 6, pp. 3915-3921, Jun. 2013.

[21] R. Cleve (1997, Jun.). Quantum stabilizer codes and classical linear codes. Phys. Rev. A, At. Mol. Opt. Phys. [Online]. vol. 55, no. 6, pp. 4054-4059. Available: http://link.aps.org/doi/10.1103/PhysRevA.55.4054

22] D. J. C. Mackay, G. Mitchison, and P. L. Mcfadden, "Sparse-graph codes for quantum error-correction," IEEE Trans. Inf. Theory, vol. 50, no. 10, pp. 2315-2330, Oct. 2003.

23] Z. Babar, S. X. Ng, and L. Hanzo, "Reduced-complexity syndrome-based TTCM decoding," IEEE Commun. Lett., vol. 17, no. 6, pp. 1220-1223, Jun. 2013.

[24] J. Dehaene and B. De Moor (2003, Oct.). Clifford group, stabilizer states, linear and quadratic operations over GF(2). Phys. Rev. A, At. Mo. Opt. Phys. [Online]. vol. 68, no. 4, pp. 042318-1-042318-10. Available: http:// link.aps.org/doi/10.1103/PhysRevA.68.042318

5] L. Hanzo, T. H. Liew, B. L. Yeap, R. Y. S. Tee, and S. X. Ng, Turbo Coding, Turbo Equalisation and Space-Time Coding: EXIT-Chart-Aided Near-Capacity Designs for Wireless Channels, 2nd ed. New York, NY, USA: Wiley, Mar. 2011.

26] M. El-Hajjar and L. Hanzo, "EXIT charts for system design and analysis," IEEE Commun. Surveys Tuts., vol. 16, no. 1, pp. 1-27, May 2013.

27] S. Ten Brink, "Rate one-half code for approaching the Shannon limit by 0.1 dB," Electron. Lett., vol. 36, no. 15, pp. 1293-1294, Jul. 2000.

8] L. Kong, S. X. Ng, R. Maunder, and L. Hanzo, "Maximum-throughput irregular distributed space-time code for near-capacity cooperative communications," IEEE Trans. Veh. Technol., vol. 59, no. 3, pp. 1511-1517, Mar. 2010

[29] S. Ibi, T. Matsumoto, R. Thoma, S. Sampei, and N. Morinaga, "EXIT chart-aided adaptive coding for multilevel BICM with turbo equalization in frequency-selective MIMO channels," IEEE Trans. Veh. Technol., vol. 56, no. 6, pp. 3757-3769, Nov. 2007.

30] M. M. Wilde, Quantum Information Theory. Cambridge, USA: Cambridge Univ. Press, May 2013. [Online]. Available: http://arxiv.org/ abs/1106.1445

[31] P. Tan and J. Li, "Efficient quantum stabilizer codes: LDPC and LDPCconvolutional constructions," IEEE Trans. Inf. Theory, vol. 56, no. 1, pp. 476-491, Jan. 2010.

2] A. Grant, "Convergence of non-binary iterative decoding," in Proc. IEEE Global Telecommun. Conf., 2001, vol. 2, pp. 1058-1062.

33] J. Kliewer, S. X. Ng, and L. Hanzo, "Efficient computation of EXIT functions for non-binary iterative decoding," IEEE Trans. Commun., vol. 54, no. 12, pp. 2133-2136, Dec. 2006.

34] S. X. Ng, O. Alamri, Y. Li, J. Kliewer, and L. Hanzo, "Near-capacity turbo trellis coded modulation design based on EXIT charts and union bounds," IEEE Trans. Commun., vol. 56, no. 12, pp. 2030-2039, Dec. 2008.

759

D. P. Divincenzo, D. Leung, and B. Terhal, "Quantum data hiding," IEEE Trans. Inf. Theory, vol. 48, no. 3, pp. 580-598, Mar. 2002.

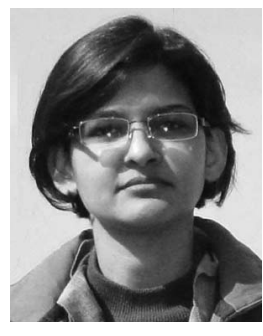

Zunaira Babar received the B.Eng. degree in elec- 760 trical engineering from the National University of 761 Science and Technology, Islamabad, Pakistan, in 762 2008 and the M.Sc. degree (with distinction) in 763 wireless communications from the University of 764 Southampton, Southampton, U.K., in 2011, where 765 she is currently working toward the Ph.D. degree 766 with the Communications, Signal Processing, and 767 Control Group, School of Electronics and Computer 768 Science.

769

Her research interests include quantum error cor- 770 rection codes, channel coding, coded modulation, iterative detection, and 771 cooperative communications.

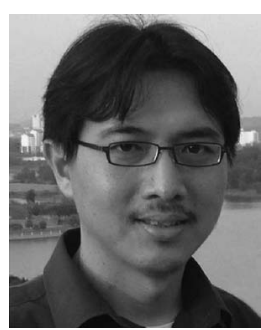

Soon Xin Ng (S'99-M'03-SM'08) received the 773 B.Eng. degree (first-class honors) in electronics en- 774 gineering and the Ph.D. degree in wireless com- 775 munications from the University of Southampton, 776 Southampton, U.K., in 1999 and 2002, respectively. 777

From 2003 to 2006, he was a Postdoctoral Re- 778 search Fellow working on collaborative European 779 research projects known as SCOUT, NEWCOM, 780 and PHOENIX. Since August 2006, he has been 781 a member of the academic staff with the School 782 of Electronics and Computer Science, University of 783 Southampton. He is involved in the OPTIMIX and CONCERTO European 784 projects, as well as the IU-ATC and UC4G projects. He is currently a Senior 785 Lecturer with the University of Southampton. He has published over 170 papers 786 and coauthored two John Wiley/IEEE Press books in his areas of interest. 787 His research interests include adaptive coded modulation, coded modulation, 788 channel coding, space-time coding, joint source and channel coding, iterative 789 detection, orthogonal frequency-division multiplexing, multiple-input multiple- 790 output, cooperative communications, distributed coding, quantum error correc- 791 tion codes, and joint wireless-and-optical-fiber communications. 792

Dr. Ng is a Chartered Engineer and a Fellow of the Higher Education 793 Academy in the United Kingdom.

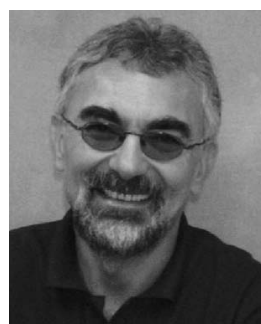

Lajos Hanzo (M'91-SM'92-F'04) received the 795 M.S. degree in electronics and the Ph.D. degree from 796 Budapest University of Technology and Economics 797 (formerly, Technical University of Budapest), 798 Budapest, Hungary, in 1976 and 1983, respectively; 799 the D.Sc. degree from the University of 800 Southampton, Southampton, U.K., in 2004; and the 801 "Doctor Honoris Causa" degree from Budapest 802 University of Technology and Economics in 2009. 803

During his 38 -year career in telecommunications, 804 he has held various research and academic posts in 805 Hungary, Germany, and the U.K. Since 1986, he has been with the School 806 of Electronics and Computer Science, University of Southampton, where he 807 holds the Chair in Telecommunications. He is currently directing a 100-strong 808 academic research team, working on a range of research projects in the field of 809 wireless multimedia communications sponsored by industry, the Engineering 810 and Physical Sciences Research Council of U.K., the European Research 811 Council's Advanced Fellow Grant, and the Royal Society Wolfson Research 812 Merit Award. During 2008-2012, he was a Chaired Professor with Tsinghua 813 University, Beijing, China. He is an enthusiastic supporter of industrial and 814 academic liaison and offers a range of industrial courses. He has successfully 815 supervised more than $80 \mathrm{Ph} . \mathrm{D}$. students, coauthored 20 John Wiley/IEEE Press 816 books on mobile radio communications totaling in excess of 10000 pages, 817 published more than 1400 research entries on IEEE Xplore, and presented 818 keynote lectures. He has more than 19000 citations. His research is funded by 819 the European Research Council's Senior Research Fellow Grant. For further 820 information on research in progress and associated publications, see http:// 821 www-mobile.ecs.soton.ac.uk.

Dr. Hanzo is a Fellow of the Royal Academy of Engineering, The Institution 823 of Engineering and Technology, and the European Association for Signal 824 Processing. He is also a Governor of the IEEE Vehicular Technology Society. 825 He has served as the Technical Program Committee Chair and the General Chair 826 of IEEE conferences, has presented keynote lectures, and has been awarded a 827 number of distinctions. During 2008-2012, he was the Editor-in-Chief of the 828 IEEE Press. 